University of Texas at El Paso

ScholarWorks@UTEP

3-1999

\title{
Chu Spaces: Towards New Foundations for Fuzzy Logic and Fuzzy Control, with Applications to Information Flow on the World Wide Web
}

\author{
Hung T. Nguyen \\ Vladik Kreinovich \\ The University of Texas at El Paso, vladik@utep.edu \\ Guoqing Liu
}

Follow this and additional works at: https://scholarworks.utep.edu/cs_techrep

Part of the Computer Engineering Commons

Comments:

UTEP-CS-99-12.

Published in Proceedings of The 18th International Conference of the North American Fuzzy Information Society NAFIPS'99, New York City, June 10-12, 1999, pp. 766-780.

\section{Recommended Citation}

Nguyen, Hung T.; Kreinovich, Vladik; and Liu, Guoqing, "Chu Spaces: Towards New Foundations for Fuzzy Logic and Fuzzy Control, with Applications to Information Flow on the World Wide Web" (1999).

Departmental Technical Reports (CS). 526.

https://scholarworks.utep.edu/cs_techrep/526

This Article is brought to you for free and open access by the Computer Science at ScholarWorks@UTEP. It has been accepted for inclusion in Departmental Technical Reports (CS) by an authorized administrator of ScholarWorks@UTEP. For more information, please contact Iweber@utep.edu. 


\title{
Chu Spaces: Towards New Foundations for Fuzzy Logic and Fuzzy Control, with Applications to Information Flow on the World Wide Web
}

\author{
Hung T. Nguyen \\ Department of Mathematical Sciences \\ New Mexico State University \\ Las Cruces, NM 88003, USA \\ hunguyen@nmsu.edu
}

\author{
Vladik Kreinovich \\ Department of Computer Science \\ University of Texas at El Paso \\ El Paso, TX 79968, USA \\ vladik@cs.utep.edu
}

\author{
Guoqing Liu \\ Department of Basic Science \\ Nanjing University of Chemical Technology \\ Nanjing 210009, P.R. China \\ lguo@dns.njuct.edu.cn
}

\begin{abstract}
We show that Chu spaces, a new formalism used to describe parallelism and information flow, provide uniform explanations for different choices of fuzzy methodology, such as choices of fuzzy logical operations, of membership functions, of defuzzification, etc.
\end{abstract}

\section{What are Chu spaces?}

\subsection{World according to classical physics}

It is well known that measurements can change the measured object: e.g., most methods of chemical analysis destroy a part of the analyzed substance; testing a car often means damaging it, etc. However, in classical (pre-quantum) physics it was assumed that in principle, we can make this adverse influence as small as possible.

Therefore, ideally, each measurement can be described as a function $r(x)$ from the set of all objects $X$ to the set $K$ of all measurement results. These measurements lead to a complete knowledge in the sense that an object $x$ can be uniquely reconstructed from the results $r(x)$ of all such measurements.

\subsection{Non-determinism in modern physics: enter Chu spaces}

In modern physics, starting from quantum mechanics, it was realized that ideal non-influencing measurements are impossible: the more accurately we measure, the more we change the object of measurement. As a result, it is not possible to uniquely reconstruct an object from measurement results. In other words, each measurement is a function $r(x, y)$ of two variables: an object $x$ and a (not completely known) measuring device $y$. Such a function describes a so-called Chu space (see, e.g., $[1,2,7,8,16,17,18,19,20,21,22]$ ).

\subsection{Precise definition of a Chu space}

To be more precise, to define a Chu space, we must fix a set $K$ (of possible values). Then, a $K$-Chu space is defined as a triple $(X, r, Y)$, where $X$ and $Y$ are sets, and $r: X \times Y \rightarrow K$ is a function which maps every pair $(x, y)$ of elements $x \in X$ and $y \in Y$ into an element $r(x, y) \in K$.

\subsection{Back to measurements: enter automorphisms of Chu spaces}

The fact that $x$ cannot be uniquely reconstructed from such measurements means that the same mea- 
surement results can be explained if we take slightly different objects $(f(x)$ instead of $x$ ) and, correspondingly, slightly different measuring instruments $(g(y)$ instead of $y): r(x, y)=r(f(x), g(y))$. This formula takes a more symmetric form if we consider, instead of $g(y)$, an inverse function $y=h(z)=g^{-1}(z)$ :

$$
r(x, h(z))=r(f(x), z) .
$$

A pair of functions $(f, h)$ which satisfies the property (1) for all $x \in X$ and $z \in Y$ is called an automorphism of a Chu space.

\subsection{From physics to general problem solving}

A general problem is: given $x$, find $y$ for which a known (easy to compute) function $r(x, y)$ takes the desired value $d$ (e.g., 0). A problem $r$ is reduced to a problem $s$ if it is possible, for each instance $x$ of the first problem, to find the correspondence instance $f(x)$ of the second problem, so that from each solution $z$ of the second problem, we can compute a solution $h(z)$ to the original problem, i.e.,

$$
r(x, h(z))=s(f(x), z) .
$$

(This notion is central in computational complexity theory, in the definitions of NP-hardness etc., see, e.g., $[5,14]$.) Such a pair $(f, h)$ is called a morphism of Chu spaces.

\subsection{Morphism of Chu spaces: precise definition}

In general, if we have two Chu spaces $A=(X, r, Y)$ and $B=\left(X^{\prime}, r^{\prime}, Y^{\prime}\right)$, the a pair of functions

$$
\left(f: X \rightarrow X^{\prime}, g: Y^{\prime} \rightarrow Y\right)
$$

is called a morphism of Chu spaces if it satisfies the property (2) for all $x \in X$ and for all $z \in Y^{\prime}$.

\subsection{Applications to parallelism and to information flow}

The notion of Chu spaces was actively used by V. Pratt (Stanford) for describing parallel problemsolving algorithms (see, e.g., [7, 8, 16, 17, 18, 19, 20, 21, 22]), and by J. Barwise (Indiana) to describe information flow in general (see, e.g., [3]).

\section{Chu spaces as a uniform justification for fuzzy techniques}

\subsection{Fuzzy is a particular case of Chu spaces}

Fuzzy knowledge can be naturally described as a Chu space $(X, r, Y)$, where $X$ is the set of all objects,
$Y$ is the set of all linguistic properties, and $r(x, y)$ is a degree to which $x$ has a property $y$ (see, e.g., [15]).

This relation was originally done in two steps:

- fuzzy logic can be interpreted as a particular case of so-called linear logic (see, e.g., [6, 11, 12, 15]), and

- linear logic is naturally interpreted in terms of Chu spaces.

\subsection{What we are planning to do}

We show that Chu description leads to a uniform justification of numerous choices of fuzzy membership functions, fuzzy logic operations, defuzzification procedures, etc. This justification is in line with a general group-theoretic approach described in our 1997 Kluwer book [13] (see also [4, 10]).

\section{The main technical idea behind using Chu spaces as a foundation for fuzzy theory: a simplified (non-fuzzy) illus- tration}

\subsection{Example: a simple physical problem}

To better present our main technical idea, we will first illustrate it on a simplified (crisp) example. Let us analyze how the period $t$ of a pendulum depends on its length $l$.

From the purely mathematical viewpoint, this dependency can be described by a function of one variable $t=F(l)$, i.e., as a function from real numbers to real numbers. However, from the physical viewpoint, such a mathematical description is somewhat unnatural, for the following reason:

- we really want a dependence between physical quantities $t$ and $l$;

- in order to describe this dependence as a dependence between real numbers, we must fix some units for measuring both length $l$ and time $t$; thus, the resulting function depends on the specific choice of these units;

- however, the choice of the units is a matter of convention (e.g., to describe length, we can use meters or feet without changing any physical meaning).

It is therefore desirable to have a mathematical description of the dependency of $t$ on $l$ which would reflect the physical dependency without adding any arbitrariness. 


\subsection{A more adequate mathematical description of the physical problem}

Such a description can be obtained if we explicitly add the two measuring units $u_{l}$ (for length) and $u_{t}$ (for time) to the description of this function, i.e., if we consider the function of the type $t=F\left(l, u_{l}, u_{t}\right)$, where $l$ is a numerical value of the pendulum's length, $t$ is a numerical value of its period, and $u_{l}$ and $u_{t}$ are the measuring units used to describe the corresponding numerical values (described in terms of some standard measuring units).

If we know the dependence $t_{0}=F\left(l_{0}\right)$ in standard units, then we can easily describe the new function: Indeed, if we use the length $u_{l}$ as a unit of length, then in these units, the numerical value $l$ of length means $l_{0}=l \cdot u_{l}$ in the original units, so in the standard units, the pendulum's period is equal to $t_{0}=F\left(l_{0}\right)=$ $F\left(l \cdot u_{l}\right)$. Hence, if we use the unit $u_{t}$ for measuring time intervals, then in this unit, the numerical value of the time period is equal to $t=t_{0} / u_{t}=F\left(l \cdot u_{l}\right) / u_{t}$. In other words, we get $F\left(l, u_{l}, u_{t}\right)=F\left(l \cdot u_{l}\right) / u_{t}$.

\subsection{Mathematical model naturally reformulated as a Chu space}

The above physically appropriate dependence can be naturally described as a Chu space, with $X$ being the set of all possible units of length, $Y$ the set of all possible time units, $K$ the set of all possible functions of one real variable, and the function $r\left(u_{l}, u_{t}\right)$ defined as $\left(r\left(u_{l}, u_{t}\right)\right)(l)=F\left(l, u_{l}, u_{t}\right)$. From the mathematical viewpoint, the sets $X$ and $Y$ coincide with the set $R^{+}$ of all positive real numbers.

\subsection{Unit-invariance formulated in precise terms}

Let us now formalize the requirement that this dependence be independent on the choice of the units for measuring length $l$ and time $t$. If we simply change a measuring unit for length or a measuring unit for time, then we get a different numerical dependence. However, for every change of the length unit, there is an appropriate change of a time unit after which the resulting numerical dependence stays the same. This requirement can be formulated as follows.

Suppose that we use a different standard unit for measuring length. Let $\lambda>0$ be the value of the old standard unit in terms of the new one; then, 1 old standard unit $=\lambda$ new standard units, so $u_{l}$ old standard units $=u_{l} \cdot \lambda$ new standard units, i.e., the measuring unit for length whose value was $u_{l}$ in old standard units has a new value $u_{l}^{\prime}=\lambda \cdot u_{l}$ in new standard units. Similarly, the choice of a new standard unit for time means that we replace the original value $u_{t}$ by a new value $u_{t}^{\prime}=g\left(u_{t}\right)$, where $g(y)=\mu \cdot y$ and $\mu$ is the value of the old standard unit in terms of the new standard unit for time.

In these terms, the above requirement means that for every function $f: X \rightarrow X$ of the type $f\left(u_{l}\right)=\lambda \cdot u_{l}$, there exists a function $g(y)$ of the type $g\left(u_{t}\right)=\mu \cdot u_{t}$ for which, for every $x \in X$ and $y \in Y$, we have $r(x, y)=$ $r(f(x), g(y))$.

\subsection{Unit-invariance reformulated in terms of Chu spaces}

We have already mentioned that this equality describes an automorphism of the Chu space. Thus, the above requirement means that for every function $f: X \rightarrow X$ from a certain transformation class can be extended to an automorphism $(f, h)$ of the corresponding Chu space.

\subsection{The Chu-space requirements describes the de- sired function}

One can show that this condition is satisfied only by functions of the type $t=A \cdot l^{\alpha}$, with $A$ and $\alpha$ arbitrary constants; the actual pendulum corresponds to $\alpha=-0.5$.

Thus, the Chu space requirement leads to a description of a very narrow class of functions which contain the desired one.

\section{Application of our main idea to fuzzy techniques: illustration and other re- sults}

\subsection{General idea}

There exist several methods of eliciting fuzzy values from experts, and, in general, different elicitation methods lead to different results. In other words, different methods may result in values corresponding to different scales of uncertainty, just like measuring the length in feet or in meters leads to different scales in which the order is preserved but numerical values are different. Similarly to the above illustrative example, it is therefore reasonable to require that the operations with fuzzy values be independent on this choice of a scale. 


\subsection{Re-scaling in fuzzy theory: an example}

One of the most natural methods to ascribe the degree of truth $d(A)$ to a statement $A$ is polling: we take several $(N)$ experts, and ask each of them whether she believes that $A$ is true. If $N(A)$ of them answer "yes", we take $d(A)=N(A) / N$. Knowledge engineers want the system to include the knowledge of the entire scientific community, so they ask as many experts as possible. But asking too many experts leads to the following negative phenomenon: when the opinion of the most respected professors, Nobel-prize winners, etc., is known, some less self-confident experts will not be brave enough to express their own opinions, so they will either say nothing or follow the opinion of the majority.

How does their presence influence the resulting uncertainty value? After we add $M$ experts who do not answer anything when asked about $A$, the number of experts who believe in $A$ is still $N(A)$, but the total number of experts is bigger $(M+N)$. So the new value of $d(A)$ is $d^{\prime}(A)=N(A) /(N+M)=c \cdot d(A)$, where we denoted $c=N /(M+N)$. From mathematical viewpoint, this transformation is exactly the same as when we use a different measuring unit in physical measurements.

\subsection{Selecting a hedge: an example of using Chu spaces}

How can we describe a hedge, i.e., an operation which transforms a degree of truth in a statement $A$ into a degree of truth for a statement "very $A$ " or "slightly $A$ "? From the purely mathematical viewpoint, we can describe this transformation as a function which transforms a numerical value $d$ of the original degree into the numerical value of the hedged degree $d^{\prime}=H(d)$. However, the exact numerical type of this function would depend on the scales use to represent both degrees. It is therefore desirable to get a representation which is independent on the choice of these scales.

Similarly to the above illustrative example, we can achieve this representation independence if we describe the hedge function as a mapping $r(x, y)$, where $x$ is a parameter which describes the scale of original degrees, $y$ is a parameter which described the scale of the hedged degrees, and $r(x, y)$ is the description of a hedge function in the scales $x$ and $y$.

Similarly to the above example, the requirement that the hedge transformation be independent on the choice of scales means that every function $f: X \rightarrow X$ from the appropriate transformation class (of linear transformations) can be extended to an automorphism of the corresponding Chu space. As a result, we deduce that all such functions have the form $d^{\prime}=A \cdot d^{\alpha}$ for some real numbers $A$ and $\alpha$. Indeed, the original Zadeh's hedges use $\alpha=2$ for "very" and $\alpha=0.5$ for "slightly". Thus, the Chu space requirement leads to a description of a very narrow class of functions which contain the desired one.

\subsection{General results}

In general, we can also have non-linear re-scalings (see, e.g., [4, 10, 13]). It turns out that the use of these re-scalings enables us to justify all major choices of fuzzy techniques: the existing choices of membership functions, of "and" and "or" operations, of defuzzification, etc.

The mathematics is, in essence, already here: in $[4,10,13]$, we have shown that these choices can be explained by the natural symmetry requirements, and similar to the above examples, these symmetry requirements can be naturally reformulated in terms of Chu spaces.

\subsection{Two applications to the Web}

First application is to web search. We can describe web search in terms of Chu morphisms (similar to problem solving): we ask a query $x$ and we want an answer $y$ which is related in a given way to $x$, i.e., for which $r(x, y)=d$ for some known function $r$. To answer this query, we translate it from a natural language query $x$ into a formal-language query $f(x)$. We then run an appropriate search engine and get a formal answer $z$ (e.g., a set of lists generated by different search tools) which is in a proper way connected to $f(x), s(f(x), z)=d$. Finally, we must translate $z$ into the desired answer $y=g(z)$. For this answer to be correct, we must guarantee that $s(f(x), z)=r(x, g(z))$, i.e., that the pair $(f, g)$ is indeed a Chu morphism. Here, ideas similar to the ones described above lead to symmetries, and symmetries, in their turn, lead to the optimal way of merging lists (i.e., we get an optimal function $g(z)$; for details, see [23]).

Another application is an optimal prediction of web growth. In general, this growth can be described by a differential equation $d L / d t=g(L)$ for some function $g(L)$. Chu-induced symmetry requirements lead to the explanation of the empirical growth formula $g(L)=C_{1} \cdot L+C_{2} \cdot L \cdot \ln (L)[9]$, and to a more general class of models aimed at a more accurate growth description [13]. 


\section{Related future work: stability of fuzzy control}

We hope that the fact that Chu spaces provide a new uniform justification for various aspects of fuzzy methodology will help in solving important problems related to this methodology.

For example, the problem of control stability can be naturally reformulated in Chu terms: if we have a control $y$ for which, for the initial situation $x$, we get the desired asymptotic behavior $r(x, y)=d$, then, for every perturbation $f(x)$ of the initial condition, we must be able to appropriately modify control (replace $y$ by $g(y))$ and achieve the same asymptotic behavior: $r(f(x), g(y))=r(x, y)$.

\section{Acknowledgments}

This work was supported in part by NASA under cooperative agreement NCC5-209, by NSF grants No. DUE-9750858 and CDA-9522207, by the United Space Alliance, grant No. NAS 9-20000 (PWO C0C67713A6), by the Future Aerospace Science and Technology Program (FAST) Center for Structural Integrity of Aerospace Systems, effort sponsored by the Air Force Office of Scientific Research, Air Force Materiel Command, USAF, under grant number F4962095-1-0518, by the National Security Agency under Grant No. MDA904-98-1-0564, and by the Hong Kong grant RGC4138/97E.

\section{References}

[1] M. Barr, *-Autonomous Categories, Springer Lecture Notes in Mathematics, Vol. 752, Springer-Verlag, Berlin, 1979.

[2] M. Barr, The Chu construction, Theory and Applications of Categories, 2:17-35, 1996.

[3] J. Barwise and J. Seligman, The logic of distributed systems, Cambridge University Press, Cambridge, N.Y., 1997.

[4] B. Bouchon-Meunier, V. Kreinovich, A. Lokshin, and H. T. Nguyen, On the formulation of optimization under elastic constraints (with control in mind), Fuzzy Sets and Systems, 81(1):5-29, 1996.

[5] M. R. Garey and D. S. Johnson, Computers and intractability: a guide to the theory of NP-completeness, Freeman, San Francisco, 1979.

[6] J.-Y. Girard, Linear logic: its syntax and semantics, In: J.-Y. Girard, Y. Lafont, and L. Regnier, eds., Advances in Linear Logic, Cambridge University Press, Cambridge, 1995, pp. 1-42.

[7] V. Gupta, Chu Spaces: A Model of Concurrency, Ph.D. Dissertation, Stanford University, Sept. 1994.
[8] V. Gupta and V. R. Pratt, Gates Accept Concurrent Behavior, Proc. 34th Ann. IEEE Symp. on Foundations of Comp. Sci., 1993, pp. 62-71.

[9] V. Gurbaxani, "Diffusion in computing networks: Bitnet", Communications of the ACM, 1990, Vol. 33, No. 12, pp. 65-ff.

[10] V. Kreinovich et al., What non-linearity to choose? Mathematical foundations of fuzzy control, Proceedings of the 1992 International Conference on Fuzzy Systems and Intelligent Control, Louisville, KY, 1992, pp. 349-412.

[11] V. Kreinovich, H. Nguyen, and P. Wojciechowski, Fuzzy Logic as Applied Linear Logic, BUlletin for Studies and Exchanges on Fuzziness and its AppLications (BUSEFAL), 67:4-13, July 1996.

[12] H. T. Nguyen and V. Kreinovich, Fuzzy Logic, Logic Programming, and Linear Logic: Towards a New Understanding of Common Sense, Proc. NAFIPS'96, Berkeley, CA, June 20-22, 1996, pp. 546-550.

[13] H. T. Nguyen and V. Kreinovich, Applications of continuous mathematics to computer science, Kluwer, Dordrecht, 1997.

[14] C. H. Papadimitriou, Computational Complexity, Addison Wesley, San Diego, 1994.

[15] B. K. Papadopoulos and A. Syropoulos, Fuzzy sets and fuzzy relational structures as Chu spaces, In: B. K. Papadopoulos and A. Syropoulos (eds.), Current Trends and Developments in Fuzzy Logic, Proceedings of the First International Workshop, Thessaloniki, Greece, October 16-20, 1998 (to appear).

[16] V. R. Pratt, Chu Spaces and their Interpretation as Concurrent Objects, In: J. van Leeuwen (ed.), Computer Science Today: Recent Trends and Developments, Springer Lecture Notes in Computer Science, 1995, Vol. 1000, pp. 392-405.

[17] V. R. Pratt, Rational Mechanics and Natural Mathematics, Proceedings of TAPSOFT'95, Aarhus, Denmark, 1995, Springer Lecture Notes in Computer Science, 1995, Vol. 915, pp. 108-122.

[18] V. R. Pratt, The Stone Gamut: A Coordinatization of Mathematics, In: Proceedings of Logic in Computer Science Conference LICS'95, IEEE Computer Society, June 1995, pp. 444-454.

[19] V. R. Pratt, Chu spaces from the representational viewpoint, Parikh Festschrift, 1997.

[20] V. R. Pratt, Types as Processes, via Chu spaces, EXRESS'97 Proceedings, 1997.

[21] V. R. Pratt, Chu spaces as a semantic bridge between linear logic and mathematics, Theoretical Computer Science, 1998.

[22] R. Van Glabbeek and G. Plotkin, Configuration Structures, In: Proceedings of Logic in Computer Science Conference LICS'95, IEEE Computer Society, June 1995, pp. 199-209.

[23] R. R. Yager and V. Kreinovich, "On How to Merge Sorted Lists Coming from Different Web Search Tools", Soft Computing, 1999 (to appear). 